\title{
Collider implications of models with extra dimensions
}

\author{
C. Macesanu ${ }^{\mathrm{a}}$, C.D. McMullen ${ }^{\mathrm{b}}$ and S. Nandi ${ }^{\mathrm{a}}$ \\ a Department of Physics, Oklahoma State University, \\ Stillwater, Oklahoma, 78074, USA \\ ${ }^{\mathrm{b}}$ Department of Physics, Penn State Altoona University, \\ Altoona, PA, 16601, USA
}

We discuss the collider signals of large extra dimensions in which gravity as well as the SM particles propagate into the extra dimensions. These signals arise either from the production of Kaluza-Klein excitations of the SM particles and their subsequent decay, or from their off-shell exchanges. Depending on the scenario, the dominant signals are two high $p_{T}$ jets + missing energy, two high $p_{T}$ photons + missing energy and soft leptons, or a combination of photon + jet and missing energy. For the scenario in which only the gauge bosons propagate into the extra dimensions, Tevatron Run II (LHC) can observe such signals up to a compactification scale of about $2 \mathrm{TeV}(7 \mathrm{TeV})$, while for the case of universal extra dimensions, the corresponding limits are about $600 \mathrm{GeV}(3$ $\mathrm{TeV}$ ) respectively.

\section{Introduction}

Recent developements [1] in string theory have led to the construction of models in which the compactification scale of the extra dimensions can be as large as inverse $\mathrm{TeV}$ [2] or even submilimeter [3]. In this later case, due to experimental constraints, only gravity can be allowed to propagate into the extra dimensions, while for the inverse $\mathrm{TeV}$ scale, both gravity and the Standard Model particles can be in the bulk. The phenomenological implications for the models with submilimeter extra dimensions have been extensively discussed in the past few years. In this talk, we will concentrate on the implications of the inverse $\mathrm{TeV}$ scale extra dimensions. In this case, there are two major possible scenarios: only the SM gauge bosons propagate into the extra dimensions, or all the SM particles propagate in the bulk (also called the Universal Extra Dimensions scenario). A hybrid scenario [4] in which the model accomodates sub-mm (in which only gravity propagates) as well as inverse $\mathrm{TeV}$ scale extra dimensions (where SM particles also propagate) has additional interesting features. Such a

\footnotetext{
*Invited talk presented by S. Nandi at the ICHEP 2002, Amsterdam, The Netherlands, July 24-31, 2002
}

model gives rise to deviations from Newton's law of gravity at sub-mm distances and several interesting astrophysical phenomena. It also leads to new physics signals at high energy colliders due to the production of KK excitations of SM particles.

\section{Only gauge bosons in the bulk}

In this scenario, only the SM gauge bosons propagate into the extra dimensions. The SM fermions are confined to the usual $3+1$ dimensional wall, the $D_{3}$-brane. We will consider the case of only one extra dimension of inverse $\mathrm{TeV}$ size. The collider signals will be similar for more than one extra dimension. The 5 th dimension is compactified on an $S^{1} / Z_{2}$ orbifold (with $y \rightarrow-y$ symmetry) and the physical space is $M_{4} \times S^{1} / Z_{2}$, where $M_{4}$ is the Minkowski space. The five dimensional gauge fields can be expanded as:

$$
\begin{aligned}
A_{\mu}^{a} & =\frac{1}{\sqrt{\pi R}}\left[A_{\mu, 0}^{a}+\sqrt{2} \sum_{n=1}^{\infty} A_{\mu, n}^{a} \cos \left(\frac{n y}{R}\right)\right] \\
A_{4}^{a} & =\frac{\sqrt{2}}{\sqrt{\pi R}} \sum_{n=1}^{\infty} A_{4, n}^{a} \sin \left(\frac{n y}{R}\right) .
\end{aligned}
$$

Here $A_{\mu, n}^{a}$ are the $n$th KK excitations of the gauge bosons with masses $m_{n} \equiv n / R$. The collider sig- 
nals will mainly come from the production of the KK excitations of the gluons $\left(g_{n}\right)$ and their subsequent decays, or through the off-shell exchange of the KK gluons in the usual SM processes. In this model, we can have new couplings such as $\bar{q} q g_{n}, g g_{n} g_{n}, g_{n} g_{m} g_{p}$ etc. (The detailed interactions and the Feynman rules for all the allowed couplings can be found in (5). The new collider signals arise from the processes $q \bar{q} \rightarrow g^{*} \rightarrow q \bar{q}$ (dijet signal); $q \bar{q} \rightarrow g^{*} g \rightarrow q \bar{q} g$ (3-jet signal) and $q \bar{q} \rightarrow g^{*} g^{*} \rightarrow(q \bar{q})(q \bar{q})$ (4-jet signal). (Here $g^{*}$ stands for a general KK excitation of the gluon). Since these jets are coming from the decay of a very heavy particle, they carry much higher $p_{T}$ than the jets produced by the usual SM processes. The high $p_{T}$ dijet signal is observable over the usual QCD background, and gives the reach for the compactification scale of about $2 \mathrm{TeV}$ at the Tevatron Run II and $7 \mathrm{TeV}$ at the LHC [5].

\section{Universal extra dimensions}

In this scenario, all SM particles propagate in the bulk [6]. Again, for simplicity, we consider the case of only one extra dimension of inverse $\mathrm{TeV}$ size. With compactifiation on an $S^{1} / Z_{2}$ orbifold, the decomposition of the 5-dimensional gauge fields is the same as above (Eq. (1D)). In 5 dimensions, the fermion fields are vectorlike; in order to obtain the chiral fermions of the SM, there are needed two sets of fermionic fields, with different properties under the $Z_{2}$ parity. Thus, we introduce an $\mathrm{SU}(2)$ doublet, whose left-handed fields are even and right-handed fields are odd under $Z_{2}$ parity:

$$
\begin{aligned}
Q= & \frac{1}{\sqrt{\pi R}}\left\{Q_{L}+\sqrt{2} \sum_{n=1}^{\infty}\left[Q_{L}^{n} \cos \left(\frac{n y}{R}\right)\right.\right. \\
& \left.\left.+Q_{R}^{n} \sin \left(\frac{n y}{R}\right)\right]\right\}
\end{aligned}
$$

and an $\mathrm{SU}(2)$ singlet with opposite properties:

$$
\begin{aligned}
q= & \frac{1}{\sqrt{\pi R}}\left\{q_{R}+\sqrt{2} \sum_{n=1}^{\infty}\left[q_{R}^{n} \cos \left(\frac{n y}{R}\right)\right.\right. \\
& \left.\left.+q_{L}^{n} \sin \left(\frac{n y}{R}\right)\right]\right\} .
\end{aligned}
$$

With these definitions, we obtain the correct chiral nature of the SM fermions (which are build from the zero modes of the above fields). For the KK exciations we then obtain two towers for each SM fermionic field: $q_{n}^{\bullet}=Q_{L}^{n}+Q_{R}^{n}$ and $q_{n}^{\circ}=$ $q_{L}^{n}+q_{R}^{n}$ (ignoring small mixing due to Yukawa interactions). The masses of the fermionic excitations are given by $m_{n}^{2}=(n / R)^{2}+m_{S M}^{2}$. The Feynman rules for the allowed couplings in this model can be found in [7]. The most interesting feature of this model is the KK number conservation at tree level. As a consequence, excitations of gluons and quarks can only be pair produced at colliders, which results in significantly lower limits (around $350 \mathrm{GeV}$ ) set on the compactification scale $1 / R$ from current experiments [6]7].

\subsection{Decay mechanisms}

There are two possible decay scenarios for KK excitations in the UED models.

(i) Loop effects: In first order, the masses of the KK excitations of the gluons and light quarks are essentialy degenerate at each level. However, loop corrections due to virtual particles propagating in the bulk, as well as boundary terms due to the breaking of translational invariance at the orbifold boundaries will remove this degeneracy. With some assumptions (which include universality for the boundary terms as well as the introduction of a cutoff scale $\Lambda$ ) it is possible to compute the corrections to the KK masses at all levels [8]. Taking $\Lambda=10 \mathrm{TeV}$, the relative corrections to the first KK level masses are as follows: $\delta m_{g^{*}} \simeq 30 \%$, $\delta m_{q^{*}} \simeq 20 \%$ and $\delta m_{W^{*}, Z^{*}, l^{*}} \leq 10 \%$. The lightest KK particle (LKP) will then be the $\gamma^{*}$, and, due to KK number conservation, it will be stable, with all other first level excitations decaying to it. In this scenario, the decay modes for the quark and gluon excitations produced at a hadron collider will be:

$$
\begin{gathered}
g_{1} \rightarrow q \overline{q_{1}^{\bullet}}, q \overline{q_{1}^{\circ}} ; q_{1}^{\circ} \rightarrow q \gamma^{*} ; \text { and } \\
q_{1}^{\bullet} \rightarrow q Z_{1}^{*} \rightarrow q l l_{1}^{\bullet} \rightarrow q l l \gamma^{*}, \text { Br. } \sim 33 \%, \text { or } \\
q_{1}^{\bullet} \rightarrow q W_{1}^{*} \rightarrow q l^{\prime} l_{1}^{\bullet} \rightarrow q l^{\prime} l \gamma^{*}, \text { Br. } \sim 65 \% \quad(4)
\end{gathered}
$$

The final states in this scenario contains two $\gamma^{*}$, plus soft jets and leptons. If this is the only decay mode, then the observable signal will be soft leptons plus large missing energy (from the LKP). 
Then, the Tevatron Run II reach is about 300 $\mathrm{GeV}$, while the LHC reach is about $1.5 \mathrm{TeV}$ [9].

(ii) Gravity mediated decays (fat brane scenario): In this scenario, gravity-mediated interactions break KK number conservation, allowing decays such as

$g^{*} \rightarrow g G_{n}, q^{*} \rightarrow q G_{n}, \gamma^{*} \rightarrow \gamma G_{n}$

where $G_{n}$ is the KK excitation of the graviton. We assume here that gravity propagates in $N$ extra dimensions of submilimeter size (such that the above decays are kinematically allowed), while the SM particles can propagate only a small distance -inverse $\mathrm{TeV}$ size- along one of these dimensions, i.e., the SM matter lives on a fat $D_{3}$ brane [10]. In this case, one obtains a form factor describing the superposition of the matter wave function with the graviton wave function along the fifth dimension, which gives rise to the couplings which allow the decays in Eq. (5).

\subsection{Collider signals for the fat brane sce- nario}

In UED models, KK excitations of quarks and gluon are pair produced at hadron colliders. The final state signal will depend on which of the above decay mechanism, (i) or (ii), dominates. If $\Gamma_{(i i)} \gg \Gamma_{(i)}$, then the final state signal will be two jets plus missing energy due to the escaping graviton excitations. The transverse momenta of the observable jets will be quite large, as will be the missing transverse energy (for an analysis, see (7). This will allow elimination of the SM backgrounds with suitable $p_{T}$ and $\boldsymbol{E}_{T}$ cuts. The Tevatron Run II reach in this mode is about $500 \mathrm{GeV}$, while the LHC reach is about $3 \mathrm{TeV}$ [7.

If the decay mechanism (i) dominates over (ii), i.e. $\Gamma_{(i)} \gg \Gamma_{(i i)}$, then the KK excitations produced at colliders will first cascade decay to the LKP as in (伍). However, due to gravitational interaction the LKP is not stable anymore and it will decay: $\gamma^{*} \rightarrow \gamma G_{n}$. The signal in this case will the be two hard photons plus large $E_{T}$ (and some soft leptons and jets). Collider reach in this case is around $550 \mathrm{GeV}$ at the Tevatron Run II and $3 \mathrm{TeV}$ at the LHC [11].

If we are in a region of the parameter space where $\Gamma_{(i)} \simeq \Gamma_{(i i)}$, then the signal will be a mixture of the modes discussed above, that is $\gamma \gamma, \gamma+$ jet, 2 jets, plus large $\mathbb{E}_{T}$ in the final states. For details, see [1].

\section{Conclusions}

Models with extra dimensions in the inverse $\mathrm{TeV}$ range can lead to interesting phenomenology at high energy colliders. Depending on the scenario, the signals may be high $p_{T}$ dijets plus missing $E_{T}$, or even high $p_{T}$ diphotons plus $E_{T}$. Tevatron reach is from $\sim 400 \mathrm{GeV}$ to $2 \mathrm{TeV}$, while LHC reach is from $\sim 1.5 \mathrm{TeV}$ to around $7 \mathrm{TeV}$, depending on the model (the higher limits are for the case when only the SM gauge bosons propagate in the extra dimensions). For low compactification scales, LHC may observe the second level KK excitations, which will be a clear signal for the existence of extra dimensions.

\section{Acknowledgements}

This work was supported in part by the U.S. Department of Energy Grant Numbers DE-FG0398ER41076 and DE-FG02-01ER45684.

\section{REFERENCES}

1. E. Witten, Nucl. Phys. B471, 135 (1996); J. D. Lykken, Phys. Rev. D 54, 3693 (1996).

2. I. Antoniadis, Phys. Lett. B246, 377 (1990).

3. N. Arkani-Hamed, S. Dimopoulos and G. Dvali, Phys. Lett. B429, 263 (1998).

4. J. Lykken and S. Nandi, Phys. Lett. B485, $224(2000)$.

5. D.A. Dicus, C.D. McMullen and S. Nandi, Phys. Rev. D65, 076007 (2002).

6. T. Appelquist, H.-C. Cheng and B.A. Dobrescu, Phys. Rev. D64, 035002 (2001).

7. C. Macesanu, C.D. McMullen and S. Nandi, Phys. Rev. D66, 015009 (2002).

8. H.C. Cheng, K.T. Matchev and M. Schmaltz, Phys. Rev. D66, 036005 (2002).

9. H.C. Cheng, K.T. Matchev and M. Schmaltz, Phys. Rev. D66, 056006 (2002).

10. A. DeRujula, A. Donini, M.B. Gavela and S. Rigolin, Phys. Lett. B482 195 (2000); T.G. Rizzo, Phys. Rev. D64, 095010 (2001).

11. C. Macesanu, C.D. McMullen and S. Nandi, Phys. Lett. B546, 253 (2002). 